\title{
Inferior vena cava leiomyosarcoma: preoperative diagnosis and surgical management
}

\author{
Karla Elizabeth Moncayo*, Juan José Vidal-Insua, Ana Troncoso and Raúl García
}

\begin{abstract}
Inferior vena cava (IVC) leiomyosarcoma is a rare malignant neoplasm more commonly seen in women in the fifth to sixth decade of life. Complete resection of the tumor with negative margins is the only therapeutical option that has demonstrated a survival benefit. This report presents a case of a 67-year-old woman affected by a lower segment IVC leiomyosarcoma incidentally detected during a chronic abdominal pain study. The patient was treated with tumorectomy, resection and ligation of the infrarenal IVC without signs or recurrence on a 12-month follow-up.
\end{abstract}

Keywords: Inferior vena cava; Leiomyosarcoma; IVC tumor

\section{Background}

Soft tissue retroperitoneal leiomyosarcomas are rare malignant tumors that account for $0.5 \%$ of all sarcomas in adults and carry a poor outcome [1-3]. The vascular system is the most commonly affected arising from the smooth muscle cells of the inferior vena cava (IVC) in half of the cases $[1,4,5]$. It is more common in women in the fifth to sixth decade of life [1]. Diagnosis may be delayed with presenting features ranging from asymptomatic, due to their deep origin, to non-specific symptoms, due to compression, that may include palpable abdominal mass, abdominal pain, lower limb edema, thrombosis, and venous stasis [1,6]. Most information about treatment comes from case reports or small series, and complete resection of the tumor with negative margins is the only therapeutical option that has demonstrated a survival benefit [3,7-9].

We present a case of a lower segment IVC leiomyosarcoma incidentally detected during a chronic abdominal pain study. Authors and the patient as well consent to publish the information below about this case report.

\section{Case presentation}

The patient is a 67-year-old woman with relevant medical history of colon cancer treated with hemicolectomy, radiation, and chemotherapy 14 years ago without

\footnotetext{
* Correspondence: karla_moncayo@hotmail.com

Complejo Hospitalario Úniversitario de Pontevedra, Mourente s/n, Pontevedra 36071, Spain
}

recurrence during the follow-up. The patient had several visits to the emergency room for immediate postprandial abdominal pain (especially epigastric and on upper quadrants) as well as nausea, vomiting, and significant weight loss during the last 18 months. Ultrasound demonstrated thrombosis of the inferior vena cava at epigastric level with an $80 \times 30 \mathrm{~mm}$ thrombus. Computed tomography (CT scan) revealed a retroperitoneal heterogeneous soft tissue density mass that involved the infrarenal IVC without affecting the iliac veins of $8 \times 4.6 \mathrm{~cm}$ in size and no evidence of metastatic disease (Figures 1 and 2). Celiac and mesenteric arteries were patent without stenosis. A biopsy of the tumor wall was conducted through a median laparotomy, because of technical difficulties by laparoscopic approach due to previous abdominal surgery and pericava localization of the tumor. As there was no definitive intraoperative diagnosis and the patient was not informed about the therapeutical procedure, tumor extirpation was postponed until pathology results were available and so the patient can be fully informed, as well. Pathologic study demonstrated a fusocellular stromal tumor, showing fascicles which intersect at $90^{\circ}$, histochemically and immunohistochemically concordant with IVC leiomyosarcoma (LMS).

Surgical approach by transverse abdominal incision is done, and the retroperitoneal mass arising from segment I IVC was exposed through a Kocher maneuver and right colon mobilization from the renal to the iliac veins. Inflammatory tissue and fibrosis were found and dissected carefully to preserve the surrounding structures, 


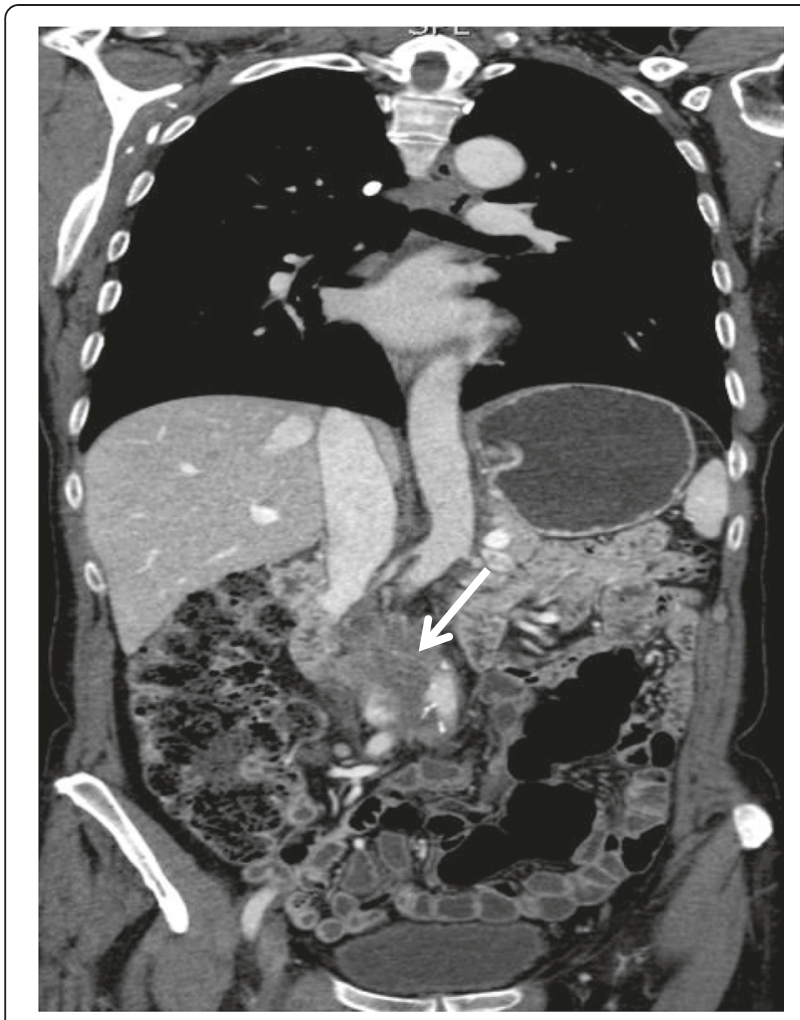

Figure 1 CT scan image. Coronal view showing retroperitoneal heterogeneous soft tissue density mass that compresses and thrombosis the infrarenal IVC of $8 \times 4.6 \mathrm{~cm}$ in size (white arrow).

especially both ureters and renal veins. Tumorectomy, resection and ligation of the infrarenal IVC, was made leaving the iliac veins patent (Figure 3).

The specimen was composed of nine pieces, the principal of $7.5 \times 3.5 \times 2.5 \mathrm{~cm}$ in diameter, diagnosed as moderately differentiated IVC LMS, with frequent areas

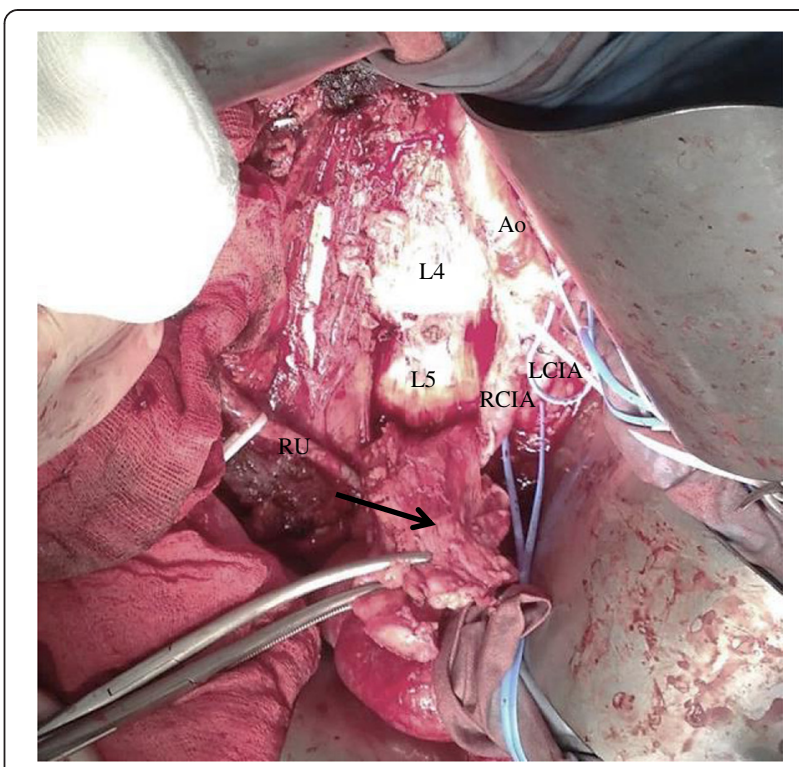

Figure 3 Intraoperative image showing inferior vena cava leiomyosarcoma during tumorectomy (black arrow). Ao, aorta; $R C I A$, right common iliac artery; $L C I A$, left common iliac artery; $L 4$, fourth lumbar vertebra; L5, fifth lumbar vertebra; RU, right ureter.

of hyalinization, foci of nuclear hyperchromatism and pleomorphism, and images of intraluminal growth (Figure 4A). Immunohistochemically, tumor cells were positive with smooth muscle markers (Figure 4B).

One week after the intervention, the patient developed an acute episode of hypotension, anemization, and severe abdominal wall hematoma due to spontaneous bleeding from epigastric collateral vessels probably secondary to anticoagulation therapy, which required reintervention for drainage and hemostasis. Postoperatively, mild lower limb edema was observed without signs of

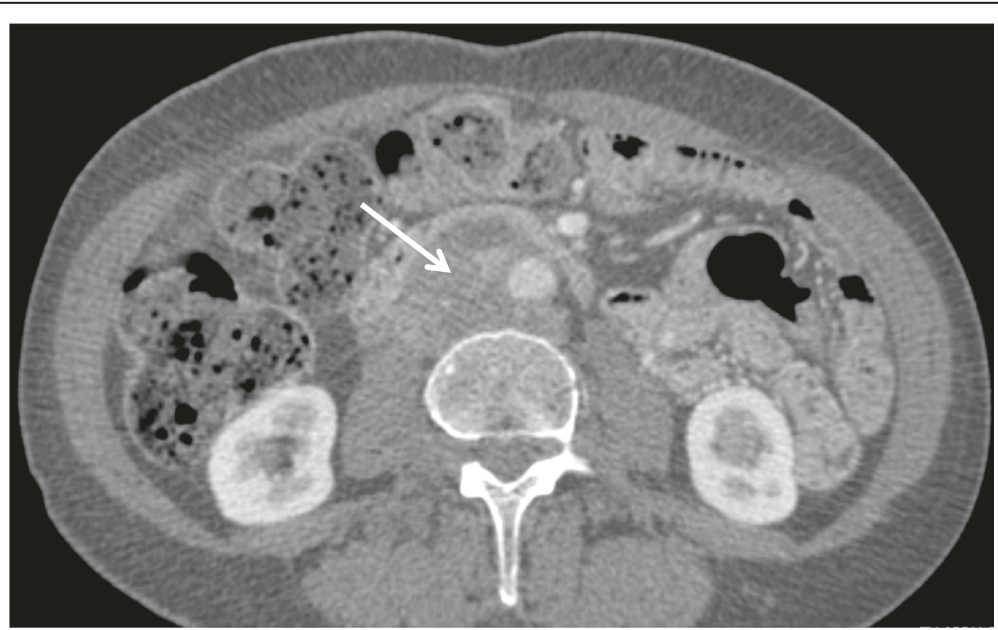

Figure $\mathbf{2}$ CT scan image. Axial view showing retroperitoneal heterogeneous soft tissue density mass that compresses and thrombosis the infrarenal IVC (white arrow). 


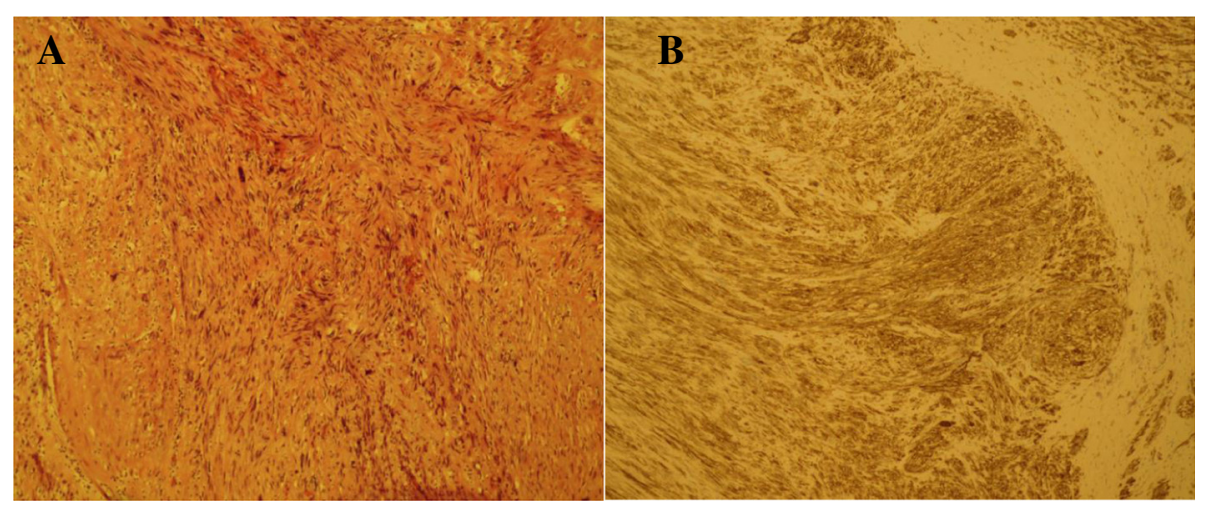

Figure 4 Inferior vena cava leiomyosarcoma histological features. (A) Microphotograph of the tumor with hematoxylin and eosin stain showing moderately differentiated IVC leiomyosarcoma, with frequent areas of hyalinization, foci of nuclear hyperchromatism and pleomorphism, and images of intraluminal growth. (B) Microphotograph of immunohistochemical actin stain, showing positive tumor cells with smooth muscle markers.

phlegmasia that improved with compression stockings. Afterwards, the clinical outcome was satisfactory, and upon discharge, the patient was anticoagulated first with low molecular weight heparin and then changed to acenocumarol with a target INR between 2 and 3 .

At 12-month follow-up, the patient has no abdominal pain, and the CT shows that there are no radiologic signs of recurrence. Meanwhile, she is on a careful follow-up program by the oncology department, and no adjuvant therapy has been performed.

\section{Discussion}

Malignant soft tissue tumors (STT) account for $<1 \%$ of malignant tumors in adults. LMS make up less than $5 \%$ of these STT, and specifically vascular LMS accounts $0.7 \%$ of all STT [10]. Two percent of LMS originates from the vascular system being IVC, the most commonly involved [1]. Retroperitoneal sarcomas are uncommon, constituting $10 \%$ to $15 \%$ of all soft tissue sarcomas (STS) with an average annual incidence of approximately 2.7 cases per million population [11]. The mean age of presentation is 53 years with a female:male ratio of $3: 1$ [12].

IVC LMS usually has a delayed clinical presentation due to slow growth and retroperitoneal location with intraluminal and/or extraluminal extension and spreading by local expansion along adjacent low-resistance structures [1,6]. Metastases are initially spread hematogenously and subsequently through lymph nodes and can involve the liver, lung, and/or bones [13]. Anatomically, IVC is divided in three segments, and according to the level affected by the tumor, the symptoms may vary. Segment I involvement (infrarenal IVC) usually presents with pedal edema and abdominal distention. Segment II (middle segment of IVC, from renal to hepatic veins) with nephrotic and/or Budd-Chiari syndrome. Segment III (above hepatic veins) with pulmonary embolism $[1,6,9]$.

Retroperitoneal LMS is more often diagnosed on CT or MRI. CT scan is the imaging modality of choice for evaluation of the tumor [14]. Coronal and sagittal slices of contrast-enhanced CT can reliably detect the origin and extension of it or if metastases are present, helping the preoperative surgical planning and determining the prognosis. Hypodense retroperitoneal mass involving the IVC with heterogeneous post contrast enhancement is the most common imaging presentation. Hence, an IVC LMS should be suspected whenever it is seen as heterogeneously enhancing as a retroperitoneal mass along the IVC $[6,14]$. Radiographic findings that indicate unresectability are peritoneal implants, distant metastases, involvement of the root of the mesentery, and spinal cord involvement [15]. In our case, we decided to confirm the diagnosis with biopsy of the tumor in order to be sure that it was not a recurrence of her previous colon cancer.

The only curative treatment that has been proved for IVC LMS is complete resection of the tumor with free margins. Adjuvant radiation and chemotherapy remains controversial and unclear $[2,4,7,12,16,17]$. Surgical options include partial resection and primary cavoplasty, complete resection and graft placement, and ligation of IVC. The latter can be performed uneventfully if a good collateral network has been developed before IVC occlusion or if it has not affected segment III IVC as it happened in our patient [3,7-9].

It has been described by Ferrario et al. on a study of 130 patients with retroperitoneal STS that local recurrence rate after local excision is $63 \%$ and after wide excision $39 \%(P<.02)$. The estimated 5 - to 10 -year survival with local recurrence was $54 \%$ and $39 \%$, respectively, and without local recurrence $66 \%$ and $58 \%$, respectively 
$(P<.05)$. The overall estimated 5-year survival from the first surgery was $60 \%$ and 10 -year survival $48 \%$. Prognostic factors for survival were the grade $(P<.001)$ followed by the procedure, wide versus local excision $(P<.01)$, whereas tumor size was not significant [18]. According to Italiano et al. on a study of 1,472 patients with sarcoma, vascular origin is an independent adverse prognostic factor for metastasis-free survival and overall survival [19]. Meanwhile, Laskin et al. on a study of 40 patients with IVC LMS demonstrated that level III IVC and right atrial involvement by sarcoma, intraluminal growth, compromised liver, and residual postsurgical macroscopic disease negatively impact on clinical course of the disease [12].

\section{Conclusions}

An enhanced retroperitoneal heterogeneous mass along the IVC should be a warning, and consideration of diagnosis of IVC LMS and an aggressive resection of it should be made as it is the only treatment that has proven survival benefits.

\section{Consent}

Written informed consent was obtained from the patient for publication of this case report and any accompanying images. A copy of the written consent is available for review by the Editor-in-Chief of this journal.

\section{Abbreviations}

CT: computed tomography; IVC: inferior vena cava; LMS: leiomyosarcoma; MRI: magnetic resonance image.

\section{Competing interests}

The authors declare that they have no competing interests.

\begin{abstract}
Authors' contributions
KEM participated in the design, coordination, and sequence alignment and drafted the manuscript. JJV helped to coordinate, design, and draft the manuscript and has given final approval of the version to be published. AT has been involved in the diagnostic pathology studies and critically revised the draft. RG has been involved in the draft and participated in revising it critically for important intellectual content. All authors read and approved the final manuscript.
\end{abstract}

\section{Authors' information}

KM is a vascular surgery fellow. JV is a vascular surgery faculty member. AT is a pathology faculty member. RG is a vascular surgery faculty member and Chairman of Angiology and Vascular Surgery Department of Complejo Hospitalario Universitario de Pontevedra, Spain

\section{Acknowledgements}

We thank Dr. Lara Alberte, a pathology faculty member, for the initial pathologic diagnosis of the patient of this case report.

Received: 3 December 2014 Accepted: 24 March 2015

Published online: 10 April 2015

\section{References}

1. Lovisetto F, Corradini C, De Cesare F, Geraci O, Manzi M, Emidi R, et al. Leiomyosarcoma of the inferior vena cava incidentally detected. Ann Vasc Surg. 2013;27(6):803. e15-9. doi:10.1016/j.avsg.2012.10.015.
2. Ito H, Hornick JL, Bertagnolli MM, George S, Morgan JA, Baldini EH, et al. Leiomyosarcoma of the inferior vena cava: survival after aggressive management. Ann Surg Oncol. 2007;14(12):3534-41. Epub 2007 Sep 25.

3. Dull BZ, Smith B, Tefera G, Weber S. Surgical management of retroperitoneal leiomyosarcoma arising from the inferior vena cava. J Gastrointest Surg. 2013;17:2166-71. doi:10.1007/s11605-013-2385-0.

4. Mann GN, Mann LV, Levine EA, Shen P. Primary leiomyosarcoma of the inferior vena cava: a 2-institution analysis of outcomes. Surgery. 2012;151 (2):261-7. doi:10.1016/j.surg.2010.10.011.

5. Xu TB, Liu WY, Chen G, Wang HZ, Bie P. Primary leiomyosarcoma of the inferior vena cava: a case report. Ann Biol Clin. 2013;71(3):338-40.

6. Naphade PS, Raut AA, Hira P, Vaideeswar P, Vadeyar H. Leiomyosarcoma of the inferior vena cava. Arch Iran Med. 2014;17(5):383-7. doi:0141705/AIM.0014.

7. Fiore M, Colombo C, Locati P, Berselli M, Radaelli S, Morosi C, et al. Surgical technique, morbidity and outcome of primary retroperitoneal sarcoma involving inferior vena cava. Ann Surg Oncol. 2012;19(2):511-8. doi:10.1245/ s10434-011-1954-2

8. Biswas S, Amin A, Chaudry S, Saju J. Leiomyosarcoma of the inferior vena cava - radical resection, vascular reconstruction, and challenges: a case report and review of relevant literature. World Journal of Oncology. 2013;4(2):107-13. http://dx.doi.org/10.4021/wjon471w.

9. Al-Saif $\mathrm{OH}$, Sengupta B, Amr S, Meshikhes AW. Leiomyosarcoma of the infra-renal inferior vena cava. Am J Surg. 2011;201(2):18-20. doi:10.1016/j. amjsurg.2010.03.021.

10. Tilkorn DJ, Hauser J, Ring A, Goertz O, Stricker I, Steinau HU, et al. Leiomyosarcoma of intravascular origin-a rare tumor entity: clinical pathological study of twelve cases. World J Surg Oncol. 2010;8:103. doi:10.1186/1477-7819-8-103.

11. Porter GA, Baxter NN, Pisters PW. Retroperitoneal sarcoma: a population-based analysis of epidemiology, surgery, and radiotherapy. Cancer. 2006;106:1610.

12. Laskin WB, Fanburg-Smith JC, Burke AP, Kraszewska E, Fetsch JF, Miettinen M. Leiomyosarcoma of the inferior vena cava: clinicopathologic study of 40 cases. Am J Surg Pathol. 2010;34(6):873-81. doi:10.1097/PAS.0b013e3181ddf569.

13. Lee HM, Jeong DS, Park PW, Kim WS, Sung K, Lee YT. Surgical treatment for an invasive leiomyosarcoma of the inferior vena cava. Korean J Thorac Cardiovasc Surg. 2013;46(5):373-6. doi:10.5090/kjtcs.2013.46.5.373.

14. Ganeshalingam S, Rajeswaran G, Jones RL, Thway K, Moskovic E. Leiomyosarcoma of the inferior vena cava: diagnostic features on cross-sectional imaging. Clin Radiol. 2011;66(1):50-6. doi:10.1016/j.crad.2010.08.004.

15. Jaques DP, Coit DG, Hadju SI, Brennan MF. Management of primary and recurrent soft-tissue sarcomas of the retroperitoneum. Ann Surg. 1990;212:51.

16. Chan AC, Chan SC, Yiu MK, Ho KL, Wong EM, Lo CM. Technical considerations for radical resection of a primary leiomyosarcoma of the vena cava. HPB (Oxford). 2012;14(8):565-8. doi:10.1111/j.1477-2574.2012.00485.x.

17. Kim JT, Kwon T, Cho Y, Shin S, Lee S, Moon D. Multidisciplinary treatment and long-term outcomes in six patients with leiomyosarcoma of the inferior vena cava. J Korean Surg Soc. 2012;82(2):101-9. doi:10.4174/jkss.2012.82.2.101.

18. Ferrario T, Karakousis CP. Retroperitoneal sarcomas: grade and survival. Arch Surg. 2003;138(3):248-51.

19. Italiano A, Toulmonde M, Stoeckle E, Kind M, Kantor G, Coindre JM, et al. Clinical outcome of leiomyosarcomas of vascular origin: comparison with leiomyosarcomas of other origin. Ann Oncol. 2010;21(9):1915-21. doi:10.1093/annonc/mdq039. 